\title{
Uretero-Arterio-Enteric Fistula Formation and Stent Thrombosis After Endovascular Treatment of Ureteroarterial Fistula: A Case Report and Review of Literature
}

\author{
Hailiu Yang, MD, ${ }^{1,2}$ Ricardo Rios, MD, Joseph Lombardi, MD, \\ Dean Matthew Marella, MD, and Allen D. Seftel, MD²
}

\begin{abstract}
Background: Ureteroarterial fistulas (UAFs) are rare life-threatening complications of indwelling ureteral stents. Endovascular repair of these fistulas is now commonly used but the long-term outcomes are unknown. Case Presentation: We present a 51-year-old African American female with history of cervical cancer status after a hysterectomy and radiation. She has bilateral ureteral strictures that were managed with chronic, indwelling ureteral stents. She subsequently developed a right UAF and was treated with an endovascular stent to the external iliac artery. After 2 years, she subsequently developed hematuria and hematochezia and was found to have a uretero-arterial-enteric fistula. We performed an exploratory laparotomy and repair of the fistula. The patient was subsequently managed with indwelling nephrostomy tubes and had no further episodes of bleeding.

Conclusion: To our knowledge, this is the first reported case of uretero-arterial-enteric fistula after endovascular treatment of UAF. Our experience demonstrates the need for a high index of suspicion and close surveillance after treatment for patients with UAF.
\end{abstract}

Keywords: ureteroarterial fistula, uretero-arterio-enteric fistula, endovascular stent complication

\section{Introduction}

$\mathbf{U}$ RETEROARTERIAL FISTUlas (UAFs) are rare occurrences. According to a recent review of literature, there were only 139 UAFs reported between 1899 and $2008 .^{1}$ These fistulas usually occur in the setting of at least one of the following risk factors: chronic indwelling ureteral stent, previous pelvic radiation, previous pelvic surgery, and vascular disease. A high index of suspicion is required for diagnosis; patients usually present with nonspecific hematuria and no single test alone has adequate sensitivity. We present a case of UAF initially treated with endovascular repair but required salvage open surgical intervention.

\section{Case Presentation}

The patient is a 51-year-old African American female who presented with multiple episodes of bloody output through her nephrostomy tube and colostomy. She had a total abdominal hysterectomy, loop colostomy, chemotherapy, and radiation for cervical cancer 6 years before presentation. Two years after her cancer treatment, she developed bilateral ureteral strictures that were managed with chronic indwelling ureteral stents, which we exchanged every $2-4$ months. Two years after having ureteral stents, she started experiencing intermittent painless gross hematuria and bleeding from her right ureteral orifice during stent exchanges. She had a ureteroscopy showing an area of inflammation and arterial bleeding at the pelvic brim when the ureteral stent was exchanged, suggestive of a UAF. She also had an angiogram and CT angiogram that were negative for signs of bleeding or any vascular abnormalities while the ureteral stent was in place. However, given her multiple episodes of bleeding and our high clinical suspicion for a UAF, our vascular surgeons placed a right external iliac endovascular stent. Active

${ }^{1}$ Division of Urology, Cooper University Hospital, Camden, New Jersey.

${ }^{2}$ Division of Urology, Cooper University Hospital, Cooper Medical School of Rowan University, Camden, New Jersey.

${ }^{3}$ Division of Vascular Surgery, Cooper University Hospital, Cooper Medical School of Rowan University, Camden, New Jersey.

${ }^{4}$ Cooper Medical School of Rowan University, Camden, New Jersey.

(c) Hailiu Yang et al. 2017; Published by Mary Ann Liebert, Inc. This is an Open Access article distributed under the terms of the Creative Commons Attribution License, which permits unrestricted use, distribution, and reproduction in any medium, provided the original work is properly cited. 


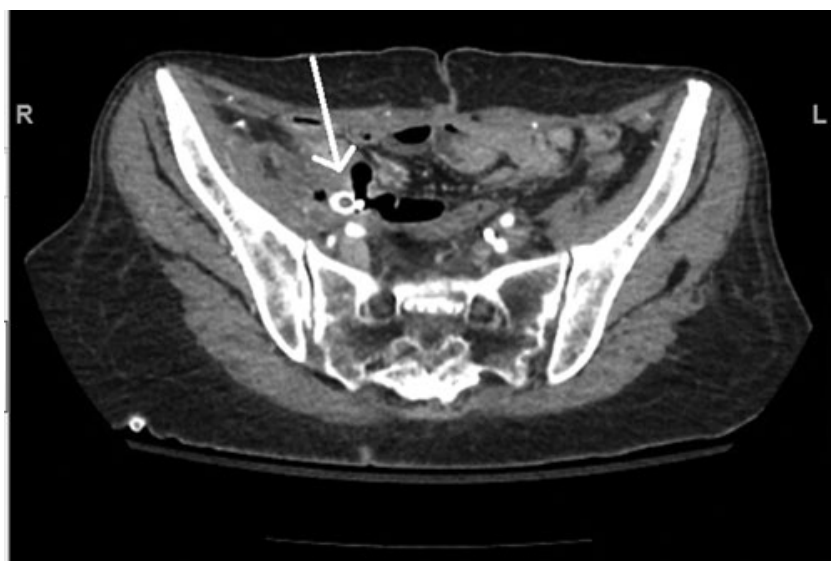

FIG. 1. CT scan of the uretero-arterio-enteric fistula. The white arrow points to the area of the fistula.

extravasation was not seen at the time of the angiogram and the indwelling ureteral stent was used as the landmark for endovascular stent deployment. The procedure was uneventful and there was no difficulty placing the endovascular stent.

Her right collecting system was subsequently managed with a nephroureterostomy catheter that was exchanged approximately every 2 months. Two years after endovascular

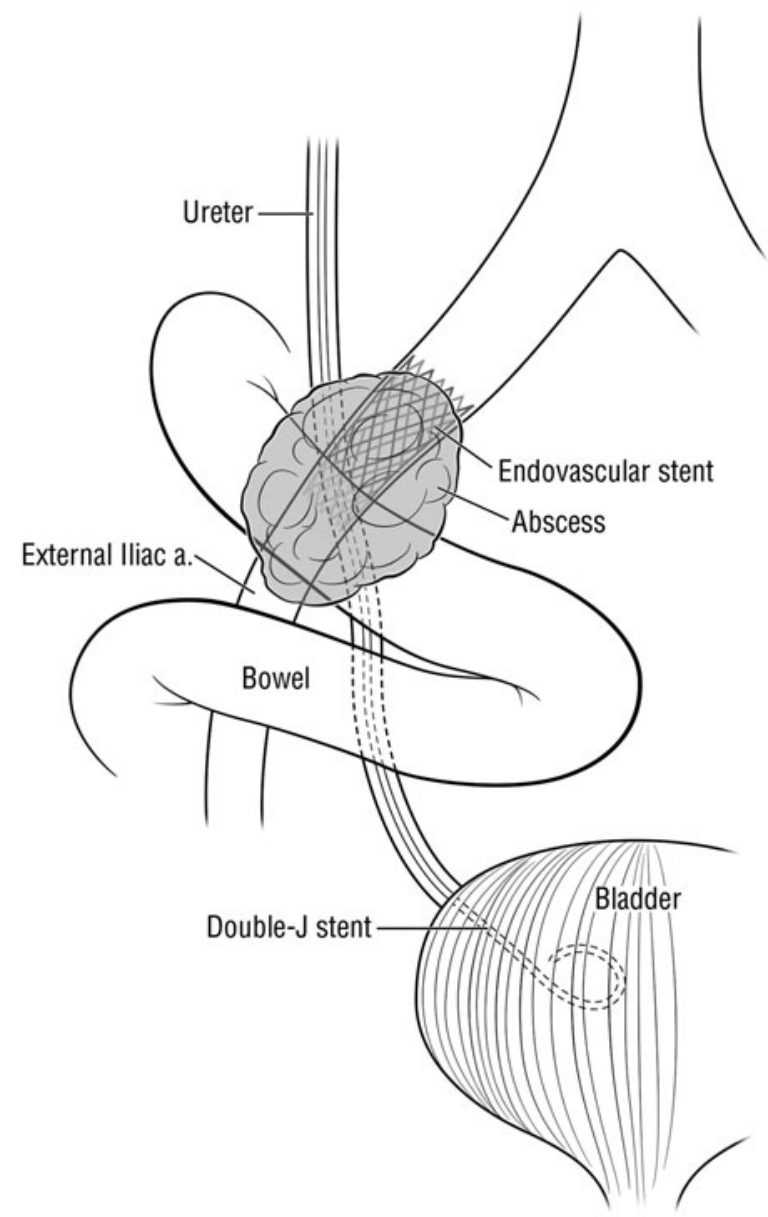

FIG. 2. Diagram of the uretero-arterio-enteric fistula.

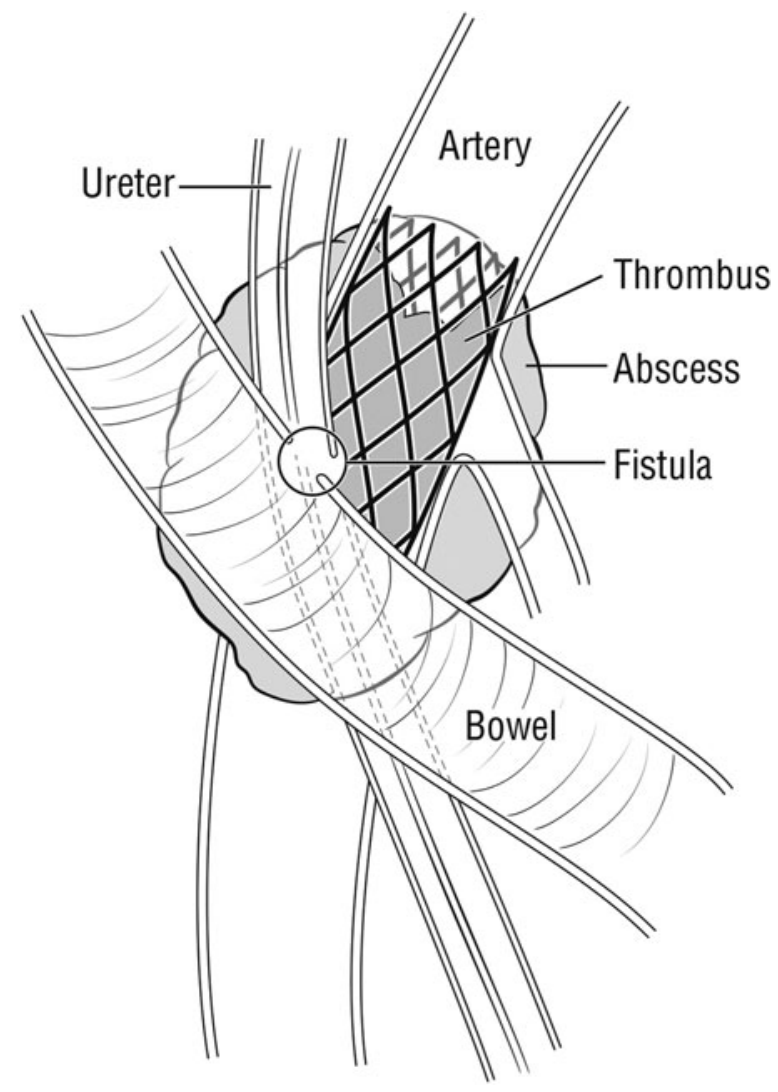

FIG. 3. Cross-section diagram of the uretero-arterioenteric fistula.

stent placement, the patient presented with bloody right nephroureterostomy tube and bloody colostomy output, requiring multiple blood transfusions. She had a negative gastroenterology work-up that included push enteroscopy, upper enteroscopy, colonoscopy, and nuclear bleeding scan. She had a CT angiogram and an MR angiogram that showed an occluded vascular stent, a complex collection of air and fluid surrounding the stent and a loop of bowel, and a large amount of air in the collecting system (Fig. 1).

These findings were highly suggestive of a three-way fistula between the small bowel, the occluded endovascular stent, and the ureter (Figs. 2 and 3). The patient was taken to the operating room for exploratory laparotomy, fistula takedown, ligation of the external iliac artery with resection of the vascular stent, ligation of the right ureter, and small bowel resection. Since the occlusion was chronic, the patient did not have evidence of limb ischemia and no revascularization was performed. The patient had no major postoperative complications. The patient was subsequently managed with chronic bilateral nephrostomy tubes that we exchanged every 6-8 weeks. We have followed the patient for 2 years after her last surgery and she had no further episodes of gross hematuria or hematochezia.

\section{Discussion}

UAFs are rare yet potentially lethal complications of indwelling ureteral stents. In a review of 139 cases from 1899 to 2008, Van den Berg reported 13\% cause-specific mortality and $100 \%$ mortality if no treatment is pursued. Diagnosis of 
this rare condition is difficult. Nonspecific hematuria was the initial symptoms in all 139 patients reported in Van den Bergh et al. ${ }^{1}$ In addition, no single diagnostic test is sensitive and specific enough; patients undergo an average of 2.4 diagnostic tests before final diagnosis. (1) CT with contrast (helpful or diagnostic in $42 \%-55 \%$ of cases) ${ }^{1,2}$ or angiogram (diagnostic or helpful in $0 \%$ to $69 \%$ of cases) $)^{1,3}$ is usually the first line tests, but their efficacy is limited because ureteral stents usually tamponade the bleeding during these tests. Provocative angiogram is more sensitive (63\% to $100 \%)$ but may cause life-threatening hemorrhages and should, therefore, only be done in a controlled setting. ${ }^{1,4}$ Cystoscopy can be useful in diagnosis by localizing the side of bleeding during stent changes. Ureteroscopy may allow the surgeon to directly observe the fistula site. Our case initially presented with sustained life-threatening bleeding episodes and received blood transfusions before diagnosis and treatment. Uretero-iliac-enteric fistula was diagnosed based on unilateral bleeding during stent exchanges and confirmed by the resolution of hematuria after the endovascular stents placement. Her CT angiogram was nondiagnostic. The patient also received numerous negative diagnostic tests for her uretero-iliac-enteric fistula before it was diagnosed. These findings again emphasize the inadequacies of diagnostic tests. In a patient with multiple risk factors and a convincing history, it is reasonable to intervene without definitive radiologic evidence.

Definite treatment involves treating both the artery and the ureter. Many methods have been reported in the literature with variable success. ${ }^{1}$ Minimally invasive endovascular treatments have recently gained popularity because patients who develop these fistulas usually have multiple comorbidities and hostile surgical abdomens from previous surgery and external radiation therapy. Although long-term patency and complication rate of endovascular procedures for aortoiliac occlusive disease are well studied and typically good, they are poorly studied in the setting of urovascular fistulas. van den Bergh and coworkers ${ }^{1}$ reported $100 \%$ fistula-specific survival rate after endovascular repair but their results were not replicated by others. Fox et al. ${ }^{4}$ reported that 2 of 14 patients who were initially treated with an endovascular procedure required open surgery at a median follow-up of only 4.5 months. Okada and coworkers ${ }^{3}$ reported a $40 \%$ hematuria-free survival and $85.7 \%$ freedom from UAF-related mortality at 2 years in a series of 11 patients (mean follow-up 1.5 years). Although there have been a few uretero-arterio-enteric fistula cases reported in the literature, this is the first reported case of this fistula after a failed endovascular repair of a UAF. We believe that maintaining an indwelling nephroureteral catheter after initial treatment, in addition to the patient's multiple risk factors for fistula formation (history of radiation, previous abdominal surgery, and vascular disease), contributed to recurrent fistula formation. Our complication serves as a reminder of the potential risks of endovascular treatment and that we need to have a high index of suspicion and close surveillance after definitive treatment for UAF.

\section{Conclusion}

Minimally invasive endovascular treatment is rapidly becoming the most popular treatment option for UAFs. However, the long-term outcome of endovascular intervention in this setting is not well studied. We present a case of failed endovascular treatment of a UAF requiring salvage open surgical intervention for a uretero-arterio-enteric fistula. Our case demonstrates that in a patient with the appropriate risk factors, we should have a high index of suspicion and close surveillance after definitive treatment.

\section{Disclosure Statement}

No competing financial interests exist.

\section{References}

1. Van den Bergh RC, Moll FL, de Vries JP, et al. Arterioureteral fistulas: Unusual suspects: Systematic review of 139 cases. Urology 2009;74:251-255.

2. Vandersteen DR, Saxon RR, Fuchs E, et al. Diagnosis and management of ureteroiliac artery fistula: Value of provocative arteriography followed by common iliac artery embolization and extra-anatomic arterial bypass grafting. J Urol 1997;156:754-758.

3. Okada T, Yamaguchi M, Muradi A, et al. Long-term results of endovascular stent graft placement of ureteroarterial fistula. Cardiovasc Intervent Radiol 2013;36:950-956.

4. Fox, JA, Krambeck A, McPhail F, et al. Ureteroarterial fistula treatment with open surgery versus endovascular management: Long-term outcomes. J Urol 2011;185:945-950.

Address correspondence to: Hailiu Yang, MD Division of Urology Cooper University Hospital 3 Cooper Plaza Suite 411

Camden, NJ 08103

E-mail: yang-hailiu@cooperhealth.edu

Cite this article as: Yang $\mathrm{H}$, Rios R, Lombardi $\mathrm{J}$, Marella DM, Seftel AD (2017) Uretero-arterio-enteric fistula formation and stent thrombosis after endovascular treatment of ureteroarterial fistula: a case report and review of literature, Journal of Endourology Case Reports 3:1, 173-175, DOI: 10.1089/cren.2017.0108. 